\title{
Prevalência de distúrbios psíquicos menores em fisioterapeutas intensivistas de uma grande cidade do estado da Bahia
}

\author{
Prevalence of minor psychic disorders in \\ intensivist physiotherapists from a large city \\ in the state of Bahia
}

\author{
Lorena Pacheco Cordeiro Lisboa' ${ }^{1}$ (]) \\ Cleide Lucilla Carneiro Santos ${ }^{2}$ (1) \\ Gabriella Bené Barbosa ${ }^{3}$
}

\section{Davi Félix Martins Junior ${ }^{4}$ Mônica de Andrade Nascimento 5 Carlito Lopes Nascimento Sobrinho ${ }^{6}$}

${ }^{1,4-6}$ Universidade Estadual de Feira de Santana (Feira de Santana). Bahia, Brasil. lorefisio@hotmail.com, dmartins2006@gmail.com, monica@uefs.br, mon.ica@terra.com.br ${ }^{2}$ Autora para correspondência. Universidade Estadual de Feira de Santana (Feira de Santana). Bahia, Brasil. kleidelucylla@hotmail.com ${ }^{3}$ Unidade de Ensino Superior de Feira de Santana (Feira de Santana), União Metropolitana de Educação e Cultura (Feira de Santana). Bahia, Brasil. gbenebarbosaster@gmail.com.br

RESUMO | INTRODUÇÃO: Os estudos sobre Distúrbios Psíquicos Menores (DPM) em fisioterapeutas intensivistas são raros, e muitos desses profissionais ainda desconhecem a relação entre o trabalho e saúde mental. OBJETIVO: Estimar a prevalência de Distúrbios Psíquicos Menores (DPM) em Fisioterapeutas intensivistas de uma grande cidade do Estado da Bahia. MÉTODOS: Estudo populacional, descritivo abrangendo 60 fisioterapeutas intensivistas que atuavam na cidade em 2016. O critério de inclusão foi trabalhar em UTI há pelo menos seis meses, para evitar o viés de trabalhador saudável. Os critérios de exclusão foram: atuar em atividade administrativa, estar em gozo de férias, em licença médica ou licença maternidade. Um questionário autoaplicável avaliou dados sociodemográficos, características do trabalho e DPM por meio do Self Report Questionnaire (SRQ-20). RESULTADOS: Dos trabalhadores estudados, 51,7\% trabalhavam em UTI adulto, 20,0\% em UTI pediátrica e $28,3 \%$ em UTI neonatal, 38,4\% dos profissionais estudados trabalhavam em duas ou mais unidades. O sexo feminino predominou, com $80,0 \%$ dos trabalhadores estudados, a média de idade foi de $32,2 \pm 4,9$ anos, $45,0 \%$ tinham companheiro, 58,3\% não tinham filhos. A prevalência de DPM foi de $41,7 \%$. conCLUSÃo: Observou-se elevada prevalência de DPM entre os fisioterapeutas intensivistas estudados. Os resultados apontam a necessidade de novos estudos que investiguem a relação entre trabalho e saúde mental em fisioterapeutas intensivistas.

PALAVRAS-CHAVE: Sofrimento Mental. Prevalência. Fisioterapeutas. Unidade de Terapia Intensiva.
ABSTRACT | INTRODUCTION: Studies on Minor Psychological Disorders (MPD) in intensive care physiotherapists are rare, and many of these professionals are still unaware of the relationship between work and mental health. OBJECTIVE: To estimate the prevalence of Minor Psychiatric Disorders (MPD) in intensive care physiotherapists in a large city in the state of Bahia. METHODS: Population, a descriptive study covering 60 intensive care physiotherapists who worked in the city in 2016. The inclusion criterion was working in the ICU for at least six months, to avoid the bias of a healthy worker. The exclusion criteria were: acting in administrative activity, being on vacation, on sick leave, or maternity leave. A self-administered questionnaire assessed sociodemographic data, job characteristics, and DPM through the Self Report Questionnaire (SRQ-20). RESULTS: Of the workers studied, $51.7 \%$ worked in an adult ICU, $20.0 \%$ in a pediatric ICU, and $28.3 \%$ in a neonatal ICU, $38.4 \%$ of the professionals studied worked in two or more units. The female gender predominated, with $80.0 \%$ of the workers studied, the average age was $32.2 \pm 4.9$ years, $45.0 \%$ had a partner, $58.3 \%$ had no children. The prevalence of MPD was $41.7 \%$. CONCLUSION: There was a high prevalence of MPD among the intensive care physiotherapists studied. The results point to the need for further studies to investigate the relationship between work and mental health in intensive care physiotherapists.

KEYWORDS: Mental Suffering. Prevalence. Physiotherapists. Intensive Care Unit. 


\section{Introdução}

A investigação da relação trabalho e saúde entre os trabalhadores de saúde, no Brasil, ocorreu mais lentamente do que em outras categorias profissionais. Estudos indicam o aumento na frequência de doenças, de estresse ocupacional e de sofrimento mental entre esses trabalhadores, especificamente entre os fisioterapeutas intensivistas ${ }^{1,2}$. Estudos apontam uma contribuição importante das características do ambiente de trabalho, das condições gerais da oferta e gestão do trabalho nos serviços de saúde com o sofrimento psíquico dos trabalhadores que atuam nesses serviços 3,8 .

As atividades dos fisioterapeutas intensivistas são fortemente tensionadas por longas jornadas de trabalho e pelo desgaste psicoemocional nas tarefas realizadas no ambiente da UT11,2. No Brasil, pesquisadores têm realizado estudos epidemiológicos com trabalhadores da área de saúde observando a associação entre as condições e características do trabalho em saúde e os Distúrbios Psíquicos Menores (DPM) $)^{3,4,6-11}$.

Os Distúrbios Psíquicos Menores são condições clínicas caracterizadas por alterações nos pensamentos e nas emoções ou por comportamentos relacionados à angústia pessoal e/ou à deterioração do funcionamento psíquico, tendo efeitos deletérios, atingindo não somente o indivíduo, mas a família e a comunidade $^{5}$. Os sintomas de DPM incluem esquecimento, dificuldade na concentração e tomada de decisões, insônia, irritabilidade e fadiga, assim como queixas somáticas (cefaleia, falta de apetite, tremores, má digestão, entre outros), o que pode comprometer o desempenho nas atividades laborais ${ }^{12}$.

As Unidades de Terapia Intensiva (UTI) são historicamente consideradas como importante causa de estresse para os pacientes e seus familiares. Porém, atualmente tem se destacado que esse ambiente também é estressante para a equipe profissional. Este estresse pelo trabalho em UTI ocorre principalmente por se tratar de um ambiente fechado, com condições e ritmos de trabalho extenuantes, rotinas exigentes, questões éticas que cabem decisões frequentes e difíceis, convívio com sofrimento e morte, imprevisibilidade e carga horária de trabalho excessiva2,7,13-15.
O profissional fisioterapeuta passou a ser membro integrante da equipe multidisciplinar nas UTIs, por meio da Portaria 3432/98 do Ministério da Saúde que também definiu a proporção de leitos por profissional, que não deve ultrapassar a de 01 (um) fisioterapeuta para cada 10 (dez) leitos, compondo a equipe básica de saúde junto com médicos e enfermeiros ${ }^{16}$.

A literatura registra que DPM constituem um importante fator de adoecimento entre os trabalhadores de saúde ${ }^{3-8,10,11}$, porém existem poucos estudos abordando fisioterapeutas intensivistas e muitos profissionais desconhecem esse distúrbio como fator de adoecimento no trabalho.

Diante do exposto, esse estudo tem como objetivo estimar a prevalência de Distúrbios Psíquicos Menores, por meio do Self Report Questinnaire (SRQ-20), em fisioterapeutas intensivistas de uma grande cidade do Estado da Bahia.

\section{Metodologia}

Trata-se de um estudo populacional, descritivo, abrangendo todos os fisioterapeutas intensivistas que atuavam na cidade no ano de 2016. Este estudo representa um recorte do projeto "Saúde Mental de Trabalhadores Intensivistas de uma Grande Cidade da Bahia", elaborado e executado por pesquisadores da Sala de Situação e Análise Epidemiológica e Estatística (SSAEE). O estudo foi aprovado pelo Comitê de Ética em Pesquisa da Universidade Estadual de Feira de Santana (CEP/UEFS), CAAE 49119315.4.0000.0053, cumprindo, dessa forma, as determinações da Resolução 466/2012 $2^{12}$.

Foi estudada a população de fisioterapeutas intensivistas, que, no ano da realização do estudo, totalizavam 60 trabalhadores. Esses fisioterapeutas trabalhavam em sete (07) dos oito (08) hospitais que tinham Unidade de Terapia Intensiva, e foram incluídos no estudo após a autorização da participação dos mesmos pela direção dos hospitais. Ressalta-se que a direção de um 01 hospital não autorizou a pesquisa, porém, os fisioterapeutas intensivistas que trabalhavam nessa unidade foram pesquisados, pois também trabaIhavam em outros hospitais da cidade. Dentre as unidades incluídas no estudo: um (01) hospital geral de 
referência em urgência e emergência da macrorregião Centro-leste da Bahia, um (01) hospital estadual de referência em atendimento pediátrico, um (01) hospital municipal e quatro (04) hospitais da rede privada, sendo uma (01) maternidade, um (01) de referência em cardiologia, e dois (02) de urgência/emergência (adulto/pediátrico).

Foram considerados elegíveis todos os fisioterapeutas $(n=60)$ que atuavam em terapia intensiva, cadastrados no setor de Recursos Humanos dos sete hospitais investigados. O critério de inclusão foi trabalhar em UTI há pelo menos seis meses, para evitar o viés de trabaIhador saudável. Os critérios de exclusão foram: atuar em atividade administrativa, estar em gozo de férias, em licença médica ou licença maternidade.

A coleta de dados foi realizada entre julho a setembro de 2016, por meio da distribuição de questionário autoaplicável, acompanhado do Termo de Consentimento Livre e Esclarecido (TCLE). Os questionários foram acompanhados de carta de apresentação e justificativa do trabalho e encaminhados aos trabalhadores de UTI, checando-se os profissionais que devolverem os questionários pelos respectivos números de identificação (cada número de questionário correspondia a um profissional pesquisado).

Foi utilizado um questionário autoaplicável, anônimo, composto por nove blocos de questões: identificação geral; informações gerais sobre o trabalho; características psicossociais do trabalho; síndrome de estafa profissional; qualidade de vida; capacidade para o trabalho; aspectos relacionados à saúde (SRQ 20) ${ }^{5}$; hábitos de vida e padrão de sono, e fatores de estresse na UTI.

O questionário e o TCLE foram entregues pelos pesquisadores dentro de um envelope a cada trabalhador nas unidades, estabelecendo o local e a hora da devolução. Os pesquisadores esclareceram sobre os objetivos do estudo e as instruções gerais sobre o preenchimento. Os profissionais que não devolviam o questionário na data agendada eram contatados por telefone, buscando-se minimizar perdas e/ou recusas.
Os questionários foram devolvidos em envelopes lacrados para garantir o sigilo e a confidencialidade.

O SRQ foi desenvolvido por Harding et al..$^{17}$ sob a coordenação da Organização Mundial de Saúde (OMS) e validado para utilização no Brasil por Mari e Willians ${ }^{18}$, com a finalidade de suspeitar morbidade psiquiátrica em instituições primárias de saúde (não hospitalares). A versão brasileira do SRQ possui 20 questões, 04 sobre sintomas físicos e 16 sobre sintomas psicoemocionais, que apresentam respostas dicotômicas "sim" ou "não", atribuindo-se respectivamente os valores "1" e " 0 " às respostas. A validação do SRQ 20 adotou o escore de 07 ou mais respostas positivas ( $\geq 7$ ) para a suspeição de morbidade psiquiátrica (DPM), obtendo-se sensibilidade de $83 \%$ e especificidade $80 \% 17,18$.

O grau de suspeição para distúrbios psíquicos foi avaliado com base no escore alcançado por cada fisioterapeuta no SRQ-20. Adotou-se o ponto de corte igual ou maior que sete respostas positivas, como em outras pesquisas na área de saúde do trabalhador 34.9 .

Foi realizada dupla digitação dos dados coletados no programa EpiData versão 3.1, para minimizar possíveis erros. Utilizou-se o software Statistical Package for Social Science (SPSS $®$ ) para a análise estatística. A análise descritiva dos dados foi realizada com o cálculo das frequências absolutas e relativas das variáveis qualitativas e da média e do desvio padrão das variáveis quantitativas. Os resultados foram apresentados por meio de tabelas.

\section{Resultados}

Este estudo teve a participação de 60 fisioterapeutas, sendo $80 \%$ (48) do sexo feminino. A média observada para a faixa etária foi de $32,2 \pm 4,9$. Quanto à cor da pele, 53,3\% (32) se consideram pardos, 30,0\% (18) brancos, 8,3\% (05) pretos, 6,7\% (04) amarelos e 1,7\% (01) não respondeu. Em relação à situação conjugal, $55 \%$ (33) eram solteiros, 36,7\% (22) eram casados, $5,0 \%(03)$ tinham união estável, 3,3\% (02) eram divorciados e $58,3 \%$ dos fisioterapeutas não tinham filhos. 
Dentre os profissionais estudados, 76,7 \% (46) têm o título de especialista, 15,0\% (09) não tinham especialização, 6,7\% (04) têm mestrado e 1,7\% (01) tem residência. Em relação a renda, 63,3\% (38) informaram renda mensal entre $\mathrm{R} \$ 3.001,00-6.000,00,18,3 \%$ (11) renda mensal menor que $\mathrm{R} \$ 3.000,00,11,7 \%$ (7) entre $6.001-10.000,00$ e $6,7 \%$ (4) entre 10.001- 20.000,00. Entre os trabalhadores estudados, 51,7\% (31) trabaIhavam em UTI adulto, 20,0\% (12) em UTI pediátrica e 28,3\% (17) em UTI neonatal (Tabela 1).

Entre os aspectos relacionados ao trabalho, a maioria dos fisioterapeutas, 63,3\% (38), apresentavam menos de cinco anos de trabalho em UTI, e 36,7\% (22) mais que 6 anos; 56,7\% (22) trabalhavam em plantão de 24 horas e 40\% (24) em plantões de 12 horas em UTI. Em relação à carga horária semanal de plantão 65\% (39) trabalhavam de 24 a 30 horas, 30\% (18) trabalhavam de 36 a 78 horas e 5\% (3) trabalhavam 12 horas semanais. A carga horária total de trabalho semanal informada, incluindo, além dos plantões, outras atividades laborais que geram renda, 50,8\% (30) dos profissionais trabalhavam menos que 56 horas e $45,8 \%$ (27) mais que 56 horas, uma média de 57,26 de carga horária total de trabalho semanal e mediana de 55 horas.

Dentre essas atividades além da UTI, a maioria, $63,3 \%$ (38), dos fisioterapeutas trabalhavam em outra especialidade, $13,3 \%$ (8) eram docentes, $18,3 \%$ (11) não apresentavam outra atividade e 5\% (3) informaram trabalhar em outro tipo de atividade, diferente da área de saúde. Com relação ao trabalho noturno em UTI, 90\% (54) dos profissionais trabalhavam entre 12 a 24 horas e 10\% (6) trabalhavam entre 36 a 96 horas, com uma média 19,3 horas e mediana de $12 \mathrm{~h}$ de trabalho noturno. Em relação à quantidade de hospitais que esses trabalhadores atuavam, verificou-se que a maioria $61,7 \%$ (37) trabalhavam em 1 hospital, 30\%
(18) trabalhavam em 2 hospitais, 6,7\% (4) trabalham em 3 hospitais e 1,7\% (1) em até 4 hospitais.

Pôde-se verificar uma diferença quanto ao número de pacientes atendidos por plantão, a maioria dos profissionais $75 \%$ atendia 10 pacientes por plantão, $16,7 \%(10)$ atendiam 08 pacientes, 3,3\% (2) 14 pacientes, 3,3\% (2) 5 pacientes e 1,7\% (1) atendia até 15 pacientes por plantão. O vínculo Institucional mais comum entre os fisioterapeutas foi assalariado no setor privado, representado por $26,7 \%$ (16), 23,3\% (14) eram assalariados no setor público 23,3\% (14), 21,7\% (13) eram cooperativados, $13,3 \%$ (8) informaram ser prestador de serviço, 8,3\% (5) apresentavam contrato temporário e 3,3\% (2) atuavam como pessoa jurídica e $36,7 \%$ dos fisioterapeutas estudados vem de outro trabalho antes do plantão na UTI (Tabela 2).

Em relação aos hábitos de vida dos trabalhadores estudados, 58,3\% (35) não bebiam e 41,7\% (25) faziam uso de bebida alcoólica. Quanto ao hábito de fumar, $88,3 \%$ (53) nunca fumaram, 6,7\% (4) eram ex-fumantes, 1,7\% (1) fumava até 4 cigarros por dia. A prática de atividade física estava presente nos hábitos de vida de $56,7 \%$ (34) dos profissionais estudados e 43,3\% (26) não realizavam nenhuma atividade física. No entanto, entre os que praticam atividade física, $73,5 \%$ (25) apresentavam frequência de duas vezes por semana, $20,6 \%$ (7) três vezes por semana e 5,9\% (2) uma vez por semana. Desses trabalhadores, $45 \%$ (27) se considerava um pouco acima do peso ideal, $35 \%$ (21) concordam que estão com peso ideal, 11,7\% (7) acham que estão muito acima do peso e 8,3\% (5) abaixo do peso ideal (Tabela 3).

A prevalência de Distúrbio Psíquico Menor medida pelo SRQ-20, em fisioterapeutas intensivistas foi de $41,7 \%$ (25) dos trabalhadores estudados (Tabela 4). 


\begin{tabular}{|c|c|c|}
\hline $\begin{array}{c}\text { Características sociodemográficas dos } \\
\text { fisioterapeutas intensivistas }\end{array}$ & $\mathbf{N}^{*}$ & $\%$ \\
\hline \multicolumn{3}{|l|}{$\operatorname{Sexo}(N=60)$} \\
\hline Feminino & 48 & 80,0 \\
\hline Masculino & 12 & 20,0 \\
\hline \multicolumn{3}{|l|}{ Faixa Etária $(\mathrm{N}=60)$} \\
\hline$\leq 33$ anos & 36 & 60,0 \\
\hline 34 anos ou mais & 24 & 40,0 \\
\hline \multicolumn{3}{|l|}{ Cor da Pele $(N=60)$} \\
\hline Branca & 18 & 30,0 \\
\hline Amarela & 4 & 6,7 \\
\hline Parda & 32 & 53,3 \\
\hline Preta & 5 & 8,3 \\
\hline Não sabe & 1 & 1,7 \\
\hline \multicolumn{3}{|l|}{ Situação Conjugal $(N=60)$} \\
\hline Solteiro & 33 & 55,0 \\
\hline Casado & 22 & 36,7 \\
\hline União estável & 3 & 5,0 \\
\hline Divorciado (a) & 2 & 3,3 \\
\hline \multicolumn{3}{|l|}{ Filhos $(N=60)$} \\
\hline Não & 35 & 58,3 \\
\hline Sim & 25 & 41,7 \\
\hline \multicolumn{3}{|l|}{ Formação Acadêmica ( $N=60$ ) } \\
\hline Especialista & 46 & 76,7 \\
\hline Sem especialização & 9 & 15,0 \\
\hline Mestrado & 4 & 6,7 \\
\hline Residência & 1 & 1,7 \\
\hline \multicolumn{3}{|l|}{ Tipo de UTI $(N=60)$} \\
\hline Adulto & 31 & 51,7 \\
\hline Neonatal & 17 & 28,3 \\
\hline Pediátrica & 12 & 20,0 \\
\hline \multicolumn{3}{|l|}{ Renda Mensal (N=60) } \\
\hline$\leq 3.000,00$ & 11 & 18,3 \\
\hline $3.000,00-6.000,00$ & 38 & 63,3 \\
\hline $6.000,00-10.000,00$ & 7 & 11,7 \\
\hline $10.000,00-20.000,00$ & 4 & 6,7 \\
\hline
\end{tabular}




\begin{tabular}{|c|c|c|}
\hline $\begin{array}{c}\text { Características do trabalho dos fisioterapeutas } \\
\text { intensivistas }\end{array}$ & $\mathbf{N *}$ & $\%$ \\
\hline \multicolumn{3}{|l|}{ Tempo/Trabalho na UTI (anos) $(\mathrm{N}=60)$} \\
\hline$\leq 5$ anos & 38 & 63,3 \\
\hline$\geq 6$ anos & 22 & 36,7 \\
\hline \multicolumn{3}{|l|}{$\mathrm{CH}^{* *}$ de Trabalho em UTI $(\mathrm{N}=60)$} \\
\hline 24 horas & 34 & 56,7 \\
\hline 12 horas & 24 & 40,0 \\
\hline Outros & 2 & 3,3 \\
\hline \multicolumn{3}{|l|}{$\mathrm{CH}^{* *}$ Semanal de plantão em UTI $(\mathrm{N}=60)$} \\
\hline 12 horas & 3 & 5,0 \\
\hline $24-30$ Horas & 39 & 65,0 \\
\hline $36-78$ Horas & 18 & 30,0 \\
\hline \multicolumn{3}{|l|}{$\mathrm{CH}^{\star \star}$ de plantão noturno em UTI $(\mathrm{N}=60)$} \\
\hline $12-24$ Horas & 54 & 90,0 \\
\hline $36-96$ Horas & 6 & 10,0 \\
\hline \multicolumn{3}{|l|}{$\mathrm{CH}^{* *}$ total semanal $(\mathrm{n}=57)^{*}$} \\
\hline$\leq 56$ horas & 30 & 50,8 \\
\hline Maior que 56 horas & 27 & 45,8 \\
\hline \multicolumn{3}{|l|}{ Vínculo Institucional ( $\mathrm{N}=60)$} \\
\hline Assalariado privado & 16 & 26,7 \\
\hline Assalariado público & 14 & 23,3 \\
\hline Cooperativado & 13 & 21,7 \\
\hline Prestador de serviço & 8 & 13,3 \\
\hline Contrato temporário & 5 & 8,3 \\
\hline Pessoa Jurídica & 2 & 3,3 \\
\hline Sócio & 2 & 3,3 \\
\hline \multicolumn{3}{|l|}{ Atividade laboral fora da UTI ( $N=60)$} \\
\hline Fisioterapia em outra especialidade & 38 & 63,3 \\
\hline Não tem & 11 & 18,3 \\
\hline Docência & 8 & 13,3 \\
\hline Fora da área da saúde & 3 & 5,0 \\
\hline \multicolumn{3}{|l|}{ Trabalho em UTI $(N=60)$} \\
\hline 1 hospital & 37 & 61,7 \\
\hline 2 hospitais & 18 & 30,0 \\
\hline 3 hospitais & 4 & 6,7 \\
\hline 4 hospitais & 1 & 1,7 \\
\hline \multicolumn{3}{|l|}{ Pacientes por plantão $(\mathrm{N}=60)$} \\
\hline 05 pacientes & 2 & 3,3 \\
\hline 08 pacientes & 10 & 16,7 \\
\hline 10 pacientes & 45 & 75,0 \\
\hline 14 pacientes & 2 & 3,3 \\
\hline 15 pacientes & 1 & 1,7 \\
\hline \multicolumn{3}{|l|}{ Vem de outro trabalho $(n=60)$} \\
\hline $\operatorname{Sim}$ & 22 & 36,7 \\
\hline Não & 38 & 63,3 \\
\hline
\end{tabular}

* Respostas válidas excluídas as ignoradas; ${ }^{* *} \mathrm{CH}=$ Carga horária. 


\begin{tabular}{lcc}
\hline \multicolumn{1}{c}{$\begin{array}{c}\text { Características dos hábitos de vida dos } \quad \\
\quad \text { fisioterapeutas intensivistas }\end{array}$} & N* \\
\hline Bebida alcoólica (N=60) & 25 & 41,7 \\
Bebe & 35 & 58,3 \\
Não bebe & & \\
Hábito de fumar (N= 60) & 53 & 88,3 \\
Nunca Fumou & 4 & 6,7 \\
Ex-fumante & 1 & 1,7 \\
Fuma até 4 cigarros p/dia & 2 & 3,3 \\
Outros & & \\
Pratica alguma atividade física(N=60) & 34 & 56,7 \\
Pratica & 26 & 43,3 \\
Não pratica & & \\
Frequência semana (N=34) & 2 & 5,9 \\
1x na semana & 25 & 73,5 \\
2x na semana & 7 & 20,6 \\
3x na semana & & \\
Peso Corporal (N=60) & 21 & 35,0 \\
Peso ideal & 25,3 \\
Abaixo do ideal & 5 & 45,0 \\
Pouco acima do ideal & 27 & 11,7 \\
Muito acima do ideal & 7 & \\
\hline
\end{tabular}

Tabela 4. Prevalência de Distúrbio Psíquico Menor (DPM) medido pelo SRQ-20 na população de fisioterapeutas intensivistas, Bahia, 2016

\begin{tabular}{lcc}
\hline Sofrimento Mental (identificado pelo SRQ 20) & $\mathrm{N}$ & $\%$ \\
\hline Positivo & 25 & 41,7 \\
Negativo & 35 & 58,3 \\
Total & 60 & 100,0 \\
\hline
\end{tabular}

\section{Discussão}

Os fisioterapeutas intensivistas estudados são em sua maioria do sexo feminino, adultos jovens (idade $<34$ anos), solteiros, sem filhos, com tempo de até 6 anos de trabalho em UTI, renda mensal entre R $\$ 3.001,00$ a 6.000,00, carga horária de trabalho semanal de 24 a 30 horas, carga horária habitual de plantão de até 24 horas, carga horária de plantões noturnos de 12 a 24 horas. A maioria trabalhava em apenas um hospital, atendia pelo menos 10 pacientes por plantão, apresentava vínculo de trabalho assalariado (privado/público), não fazia uso de bebida alcoólica e não fumava, realizava atividade física com uma frequência de duas vezes por semana.

O perfil dos fisioterapeutas observado foi semelhante ao encontrado em outros trabalhos consultados que estudaram fisioterapeutas atuantes em UTI, predomínio do sexo feminino, solteiros ${ }^{1,2}$, e também, com o de outras categorias profissionais, idade média menor que 40 anos $^{4,7,8,11,19}$, e tempo de trabalho em UTI menor que 5 anos $s^{1,7}$.

A prevalência de DPM encontrado neste estudo foi de $41,7 \%$, resultado semelhante com o estudo de Pinhatti et al., , que apresentou uma prevalência global de DPM entre trabalhadores de enfermagem de 32,6\%, com o de

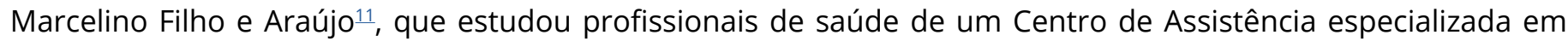
Aracaju e que estimou uma prevalência nos fisioterapeutas de $57,1 \%$ e o de Carvalho et al. $\underline{\underline{20}}$, que obteve uma prevalência de $51,1 \%$ em residentes da cidade de Recife. 
Observou-se também uma prevalência maior do que a encontrada no estudo de Nascimento et al. ${ }^{\text {, }}$ de $24,6 \%$ em enfermeiras intensivistas de Feira de Santana, Bahia, com a de Rodrigues et al. ${ }^{4}$, que estimou uma prevalência de 35,0\% em enfermeiros de um hospital geral, em Feira de Santana, Bahia; de Alves et al. ${ }^{10}$, que obteve $27,9 \%$ de positividade para DPM em profissionais de saúde do Hospital de Clínicas da Universidade Federal do Triângulo Mineiro; de Nascimento Sobrinho et al. $\frac{3}{\text {, de }}$ 26,0\% em uma amostra aleatória de médicos, em Salvador, Bahia, com a de Araújo et al. ${ }^{2}$ de 33,3\% em profissionais de enfermagem de um hospital público, em Salvador, Bahia, e com o de Carvalho, Araújo e Bernardes 5 , que encontrou uma prevalência de $22,9 \%$ em trabalhadores da Atenção Básica.

Em relação à predominância do sexo feminino, sabese há muitas décadas que o trabalho em saúde se apresenta historicamente feminino, fato que pode estar relacionado à essência das profissões de saúde, o ato de cuidar, visto como uma ação de dedicação e múltiplas funções, atribuída historicamente ao sexo feminino ${ }^{21-23}$.

Os resultados apresentados apontam que os Distúrbios Psíquicos Menores podem estar relacionados ao trabalho e, assim, podem ser considerados um problema de saúde pública, em função de sua alta prevalência em trabalhadores de saúde e de suas consequências, como o absenteísmo, incapacidade para o trabalho e aposentadoria precoce.

O trabalho em sistema de plantão noturno foi citado no estudo de Monteiro 24 como causa de estresse e de complicações à saúde, uma vez que o desequilíbrio do ritmo biológico pode gerar dificuldade na quantidade e na qualidade do sono, confirmando os achados com o estudo de Nascimento et al. ${ }^{-}$, no qual verificou-se maior prevalência de DPM entre enfermeiros intensivistas que trabalhavam à noite.

Esses distúrbios ainda não são reconhecidos com frequência nos atendimentos clínicos e setores em que as demandas psicossociais são elevadas, como as UTIs, devido às características estressantes do tipo de trabalho realizado, o que torna esses trabalhadores mais vulneráveis ao sofrimento e ao adoecimento mental ${ }^{5}$.
Em relação ao consumo de bebida alcoólica, observou-se que $41,7 \%$ dos fisioterapeutas intensivistas analisados consumiam bebida alcoólica. Em um estudo realizado com trabalhadores de enfermagem de um Hospital Geral da Bahia que utilizou o teste CAGE, instrumento de triagem para detecção de abuso no consumo de bebidas alcoólicas; dos que informaram o uso de bebida alcoólica, 27,7\% destes foram considerados bebedores-problema ${ }^{4}$, e no estudo de Nascimento et al. ${ }^{7}$, com enfermeiras intensivistas, foi encontrada associação positiva entre o consumo de bebida alcoólica e DPM, o que pode comprometer a saúde e o desempenho profissional, colocando em risco a segurança do paciente.

Com relação aos hábitos de vida, a maior parte dos fisioterapeutas, 56,7\%, relatou praticar atividade física, e dos que praticavam, 73,5\% informaram uma frequência de duas vezes por semana. A literatura aponta inúmeros benefícios relacionados com a prática da atividade física nos hábitos cotidianos e profissionais, tais como: melhora cognitiva, combate ao estresse, ansiedade, depressão, melhora das relações interpessoais e melhor desempenho nas atividades laborais ${ }^{25}$.

Importante tecer algumas considerações metodológicas desse estudo: os estudos descritivos não permitem estabelecer nexo causal, apenas descrevem a magnitude de um determinado problema de saúde, nesse caso, a prevalência de Distúrbios Psíquicos Menores e a frequência e ocorrência de certas características como sexo, idade, características do trabaIho, hábitos de vida.

Por se tratar de um estudo populacional, os resultados observados dizem respeito tão somente aos fisioterapeutas intensivistas, não sendo adequada a extrapolação dos resultados obtidos para outras realidades; a utilização do questionário autoaplicável, pela característica subjetiva do respondente, pode influenciar os resultados a depender do grau de compreensão, bem como permite a devolução de questionários com respostas incompletas. Por fim, observou-se uma escassez na literatura de estudos que abordem os DPM em fisioterapeutas que atuam em terapia intensiva, prejudicando, assim, a comparação e a discussão dos resultados observados. 


\section{Conclusão}

Os resultados apontaram elevada prevalência de DPM entre os fisioterapeutas estudados, o que sinaliza para a reflexão sobre a adoção de medidas que possam prevenir e/ou modificar os resultados encontrados. Por fim, os resultados apontam para a necessidade de novos estudos que investiguem a relação entre trabaIho e saúde mental de fisioterapeutas intensivistas.

\section{Agradecimentos}

Agradecemos ao apoio recebido da Sociedade de Terapia Intensiva da Bahia (SOTIBA - Regional da Associação Medicina Intensiva Brasileira/AMIB), do Conselho Regional de Fisioterapia e Terapia Ocupacional (CREFITO), dos estudantes da Universidade Estadual de Feira de Santana (UEFS): Adriana Mendonça da Silva, Enéias Ribeiro de Oliveira, Gabriel Silva Rocha, Jamile Prado Oliveira Santos, Karole Brito Alves Costa e Roan da Silva Gomes Sampaio e dos Enfermeiros Jailson Vieira Machado e Silvia Feitosa de Sousa que ajudaram na coleta e digitação dos dados.

\section{Contribuições dos autores}

Lisboa LPC, Santos CLC, Barbosa GB, Martins Júnior DF, Nascimento, MA e Nascimento Sobrinho CL participaram da concepção, delineamento, busca e análise estatística dos dados da pesquisa, interpretação dos resultados e redação do artigo científico.

\section{Conflitos de interesses}

Nenhum conflito financeiro, legal ou político envolvendo terceiros (governo, empresas e fundações privadas, etc.) foi declarado para nenhum aspecto do trabalho submetido (incluindo, mas não se limitando a subvenções e financiamentos, participação em conselho consultivo, desenho de estudo, preparação de manuscrito, análise estatística, etc.).

\section{Referências}

1. Silva GJP, Ferreira PAM, Costa RP, Jesus SFC, Gondim LAR, Ferreira PR. Danos à saúde relacionados ao trabalho de fisioterapeutas que atuam em terapia intensiva. Assobrafir ciência [Internet]. 2016;7(2):31-44. Disponível em: http://www.uel.br/ revistas/uel/index.php/rebrafis/article/view/25328/19676

2. Santos CLC, Barbosa GB, Nascimento DSS, Martins Júnior DF, Nascimento Sobrinho CL. Prevalência de Síndrome da Estafa Profissional e fatores associados em fisioterapeutas intensivistas. Rev Pesqui em Fisioter. 2018;8(3):336-44. Disponível em: https://www5.bahiana.edu.br/index.php/fisioterapia/article/ download/2032/1936
3. Nascimento Sobrinho CL, Carvalho FM, Bonfim TAS, Cirino CAS, Ferreira IS. Condições de trabalho e saúde dos médicos em Salvador, Brasil. Cad. Saúde Pública. 2006;22(1):131-40. https:// doi.org/10.1590/S0102-311X2006000100014

4. Rodrigues EP, Rodrigues US, Oliveira LMM, Laudano RCS, Sobrinho CLN. Prevalência de transtornos mentais comuns em trabalhadores de enfermagem em um hospital da Bahia. Rev Bras Enferm. 2014;67(2):296-301. https://doi.org/10.5935/0034$\underline{7167.20140040}$

5. Carvalho DB, Araújo TM, Bernardes KO. Transtornos mentais comuns em trabalhadores da Atenção Básica à Saúde. Rev Bras Saúde Ocup. 2016;41:e17. http://dx.doi.org/10.1590/23176369000115915

6. Pinhatti EDG, Ribeiro RP, Soares MH, Martins JT, Lacerda MR. Distúrbios psíquicos menores na enfermagem: prevalência e fatores associados. Rev Bras Enferm. 2018;71(supl 5):2176-83. http://dx.doi.org/10.1590/0034-7167-2018-0028

7. Nascimento DDSS, Barbosa GB, Santos CLC, Martins Júnior DF, Sobrinho CLN. Prevalência de Distúrbio Psíquico Menor e fatores associados em enfermeiros intensivistas. Rev Baiana Enferm. 2019;33:e28091. http://dx.doi.org/10.18471/rbe.v33.28091

8. Pascoal KPMF, Santos ACBC, Silva JASS, Fernandes VMS, Sousa MN. Avaliação da qualidade de vida, estresse e saúde mental dos profissionais de saúde das Unidade de Terapia Intensiva. Rev Interdiscip em saúde. 2019;6(5):19-30. http://dx.doi. org/10.35621/23587490.v6.n5.p19-30

9. Araújo TM, Aquino E, Menezes G, Santos CO, Aguiar L. Aspectos psicossociais do trabalho e distúrbios psíquicos entre trabalhadoras de enfermagem. Rev Saude Publica. 2003;37(4):424-33. https://doi.org/10.1590/S0034$\underline{89102003000400006}$

10. Alves AP, Pedrosa LAK, Coimbra MAR, Miranzi MAS, Hass VJ. Prevalência de transtornos mentais comuns entre profissionais de saúde. Rev Enferm Uerj. 2015;23(1):64-9. http://dx.doi. org/10.12957/reuerj.2015.8150

11. Marcelino Filho A, Araújo TM. Estresse ocupacional e saúde mental dos profissionais de Centro de Especialidades Médicas de Aracaju. Trab Educ e Saúde. 2015;13(supl 1):177-99. https://doi. org/10.1590/1981-7746-sip00016

12. Fiorotti KP, Rossoni RR, Borges LH, Miranda AE. Transtornos mentais comuns entre os estudantes do curso de medicina: prevalência e fatores associados. J. bras. psiquiatr. 2010;59(1):1723. https://doi.org/10.1590/S0047-20852010000100003

13. Barros DDS, Tironi MOS, Nascimento Sobrinho CL, Neves FS, Bitencourt AGV, Almeida ADM, et al. Médicos plantonistas de unidade de terapia intensiva: perfil sócio-demográfico, condições de trabalho e fatores associados à síndrome de burnout. Rev Bras Ter Intensiva. 2008;20(3):235-40. https://doi.org/10.1590/S0103507X2008000300005 
14. Tironi MOS, Nascimento Sobrinho CL, Barros DDS, Reis EJFB, Marques Filho ES, Almeida A, et al. Trabalho e síndrome da estafa profissional (Síndrome de Burnout) em médicos intensivistas de Salvador. Rev Assoc Med Bras. 2009;55(6):656-62. https://doi. org/10.1590/S0104-42302009000600009

15. Tironi MOS, Teles JMM, Barros DS, Vieira DFVB, Silva Filho $C M$, Martins DF, et al. Prevalência de síndrome de burnout em médicos intensivistas de cinco capitais brasileiras. Rev Bras Ter Intensiva. 2016;28(3):270-7. https://doi.org/10.5935/0103507X.20160053

16. Portaria GM/MS no 3432, de 12 de agosto de 1998 (Brasil). Estabelece critérios de classificação para as Unidades de Tratamento Intensivo - UTI. [Internet]. Gabinete do Ministro; 1998. Disponível em: http://bvsms.saude.gov.br/bvs/saudelegis/ gm/1998/prt3432_12_08_1998.html

17. Harding TW, Arango MV, Baltazar J, Climent CE, Ibrahim $\mathrm{HH}$, Ladrido-Ignacio L, et al. Mental disorders in primary health care: a study of their frequency and diagnosis in four developing countries. Psychol Med. 1980;10(2):231-41. https://doi. org/10.1017/s0033291700043993

18. Mari JJ, Willians P. A validity study of a psychiatric screening questionnaire (SRQ-20) in primary care in the city of São Paulo. Br J Psychiatry. 1986;148(1):23-6. https://doi.org/10.1192/ bjp.148.1.23

19. Koch MO, França DA, Nascimento FC, Segura DCA. Estresse físico e mental em fisioterapeutas e equipe de enfermagem em unidade de terapia intensiva. Rev Interdiscip [Internet]. 2019;12(1):23-31. Disponível em: https://revistainterdisciplinar. uninovafapi.edu.br/index.php/revinter/article/view/1390/pdf 404
20. Carvalho CN, Melo-Filho DA, Alberto J, Carvalho G, Carla A, Amorim G. Prevalência e fatores associados aos transtornos mentais comuns em residentes médicos e da área multiprofissional. J Bras Psiquiatr. 2013;62(1):38-45. https://doi. org/10.1590/S0047-20852013000100006

21. Guerrer FJL, Bianchi ERF. Caracterização do estresse nos enfermeiros de unidades de terapia intensiva. Rev Esc Enferm USP. 2008;42(2):355-62. https://doi.org/10.1590/S0080$\underline{62342008000200020}$

22. Borges T, Bianchin MA. Qualidade de vida dos profissionais de enfermagem de um hospital universitário do interior de São Paulo. Arq Ciênc Saúde. 2015;22(1):53-8. https://doi. org/10.17696/2318-3691.22.1.2015.29

23. Machado $\mathrm{MH}$, Aguiar Filho W, Lacerda WF, Oliveira E, Lemos $W$, Wermelinger $M$, et al. Características gerais da enfermagem: o perfil sócio demográfico. Enferm. Foco [Internet]. 2015;6(1/4):11 7. Disponível em: http://biblioteca.cofen.gov.br/wp-content/ uploads/2016/07/Caracter\%C3\%ADsticas-gerais-da-enfermagemo-perfil-s\%C3\%B3cio-demogr\%C3\%A1fico.pdf

24. Monteiro JK, Oliveira ALL, Ribeiro CS, Grisa GH, Agostini N. Adoecimento Psíquico de Trabalhadores de Unidades de Terapia Intensiva. Psicol ciênc prof. 2013;33(2):366-79. https://doi. org/10.1590/S1414-98932013000200009

25. Freire CB, Dias RF, Schwingel PA, França EET, Andrade FMD, Costa EC, et al. Qualidade de vida e atividade física em profissionais de terapia intensiva do sub médio São Francisco. Rev Bras Enferm. 2015;68(1):21-6. https://doi.org/10.1590/00347167.2015680104p 